\title{
El debate en ciencias: gana el equipo que mejor argumente
}

Esther Cascarosa Salillas. Universidad de Zaragoza

María García Andreu. IES Santiago Hernández (Zaragoza)

Jorge Pozuelo Muñoz. Universidad de Zaragoza
0000-0002-3696-7673

(iD) $0000-0002-9223-6832$

\section{Recepción: 30.12.2018 | Aceptado: 04.01.2019}

Correspondencia a través de ORCID: Esther Cascarosa Salillas

Citar: Cascarosa, E., Garcia, M. y Pozuelo, J. (2019). El debate en ciencias: gana el equipo que mejor argumente. REIDOCREA, 9, 15-20. [ ]

Agradecimientos: Proyecto MINECO EDU2016-76743-P, Grupo BEAGLE y al Instituto Universitario de Investigación en Ciencias Ambientales de Aragón -IUCA-

Resumen: La argumentación oral es una competencia científica clave que debe trabajarse en la Educación Secundaria Obligatoria a través de la práctica. Una manera de trabajarla es mediante la realización de debates en clase. Para potenciar dicha competencia se ha realizado un debate en una clase de Física y Química de $2^{\circ}$ de E.S.O. sobre el uso de la energía nuclear. Los argumentos expuestos por los alumnos para defender su postura a favor o en contra de dicho tipo de energía, se han recogido mediante grabaciones de audio y vídeo para su posterior análisis. Tras establecer una clasificación del nivel de calidad de estos argumentos, se ha proclamado el grupo vencedor del debate.

Palabras clave: Competencia argumentativa | Debate

The science discussion: the team with the best argument wins

Abstract: The oral argument is a key scientific competence that should be practiced in Compulsory Secondary Education. One way of practicing it is carrying out discussions in class. In order to improve this competence, a debate on the use of nuclear energy was carried out in a Science classroom. The arguments put forward by the students to defend their stance for or against this type of energy, were collected by means of audio and video recordings and have been studied in depth. After establishing a classification of the quality of these arguments, the winning group was announced.

Keywords: Argumentative competence | Discussion

\section{Introducción}

Dentro de la amplia gama de temas trabajados en la clase de ciencias en la Educación Secundaria Obligatoria (E.S.O.), existen algunos con la capacidad de incidir especialmente en el interés del alumnado. Este interés va más allá de la motivación debido a que son temas en los que existen relaciones intrínsecas entre ciencia y sociedad, de ahí que puedan entenderse como CTS (Ciencia, Tecnología y Sociedad). Uno de los más controvertidos, y que siempre ha estado presente en el debate social, es el de la energía nuclear. Actualmente es patente la falta de motivación en gran parte del alumnado de E.S.O. hacia las ciencias, por lo que es de relevancia aprovechar el interés que presentan temáticas como esta para trabajar las competencias científicas. Los resultados de estudios realizados a adolescentes europeos muestran niveles moderados de autoconfianza en el aprendizaje de las ciencias y bajo interés por éstas (George, 2006). Este desinterés y falta de motivación desemboca en una tendencia descendente en las actitudes hacia las ciencias en la E.S.O. (Barmby, Kind y Jones, 2008). Si estos aspectos no se tienen en cuenta, y por lo tanto no se trabajan, la consecuencia es que muchos alumnos, no solo no disfrutan de la ciencia, sino que no prosiguen sus carreras científicas o ni siquiera consiguen una adecuada alfabetización científica, deseable para toda la ciudadanía. Según Solbes, Monstserrat y Furió (2007), en la enseñanza actual de las ciencias, 
mayoritariamente se está dejando a un lado esa falta de motivación del alumnado y, sin embargo, sabemos que la autoconfianza y la motivación son dos de los aspectos más vinculados al rendimiento escolar en ciencias (Gil-Flores, 2012). Esta motivación puede lograrse dándoles un papel más activo en clase, lo que destacan como necesidad Campanario y Moya (1999). Por lo tanto, si trabajamos temas que a priori inciden en la motivación y participación activa del alumnado, facilitamos el camino para trabajar competencias científicas tan necesarias hoy en día y que son el punto de partida para seguir ampliando la formación de los alumnos en trabajos de investigación (Ferrés, Marbá y Sanmartí, 2014).

Una de estas competencias es la argumentación científica. Driver, Newton y Osborne (2000) describían la importancia de iniciar a los estudiantes en la argumentación en ciencias y la necesidad de darle un lugar clave a la formación en esta competencia (Erduran y Jiménez Aleixandre, 2007). Algunos autores han encontrado que los estudiantes de E.S.O. poseen pocas habilidades argumentativas en general. Solbes, Ruíz y Furió (2010) estudiaron esta competencia en un grupo de estudiantes de $3^{\circ}$ E.S.O., $4^{\circ}$ E.S.O. y $1^{\circ}$ de bachillerato, destacando que el alumnado utiliza poco el conocimiento científico para justificar o fundamentar sus razonamientos, concluyendo con carácter general, el bajo nivel de la competencia argumentativa, siendo ésta además menor en la argumentación oral que en la escrita. En el aula de ciencias en particular, y en la enseñanza en general, la expresión oral es decisiva porque el aprendizaje se demuestra en gran medida a través del lenguaje hablado (Jiménez Aleixandre y Díaz de Bustamante, 2003). Tras aplicar durante tres cursos escolares estrategias (como debates) para mejorar dicha competencia, los mismos autores concluyeron en una mejoría de habilidades argumentativas orales en física y química de los alumnos (Ruiz, Solbes y Furió, 2013).

En base a todo lo expuesto, parece que el desarrollo de un debate en clase, es la metodología adecuada para trabajar el tema de la energía nuclear ya que da la oportunidad de desarrollar entre otras, la capacidad de razonar y argumentar (Jiménez Aleixandre, 1998; Sardá y Sanmartí, 2000). De esta manera, los alumnos permanecen motivados y los que normalmente se muestran pasivos aumentan su participación (Solbes, Ruiz y Furió, 2010). Y, por otro lado, hacerlo de manera continuada favorece la adquisición de la competencia argumentativa. En el presente trabajo se expone una experiencia llevada a cabo en $2^{\circ}$ E.S.O. en la que se analizan los razonamientos argumentativos orales que presentan estos alumnos ante un debate sobre la energía nuclear, evaluando la calidad de dichos argumentos.

\section{Métodos}

\section{Contexto}

La propuesta se realiza en $2^{\circ}$ de ESO, dentro de la materia Física y Química. El grupo está formado por 25 estudiantes ( 15 chicas y 10 chicos). En líneas generales muestran interés por la ciencia, aunque también encontramos alumnado más desmotivado. Habitualmente trabajan las ciencias mediante una metodología tradicional en la que el alumno es mero receptor del conocimiento del profesor sin contar con un papel activo dentro de las clases. Tampoco se realizan debates para trabajar las habilidades argumentativas.

Para desarrollar la actividad, se propuso a los alumnos que formaran dos grupos de manera que hubiera uno que argumentara a favor de la energía nuclear y otro en contra. Para ello, se dejó que los alumnos se posicionaran libremente en el grupo que 
ellos consideraran. Los dos grupos formados tenían un número similar de estudiantes (12 y 13 respectivamente).

A continuación, se les explicó el sistema de trabajo que se iba a seguir. Antes de comenzar el debate, actividad principal en esta investigación, tuvieron lugar dos pasos previos. En primer lugar, se les expuso un vídeo preparado previamente, en el que se explica en qué consiste la energía nuclear. Además, aparecen datos concretos relativos a su generación y consumo (cantidad de recursos consumidos, porcentaje que supone la energía nuclear sobre el total de la energía consumida en España, etc.). Durante la visualización del video los alumnos pidieron tomar anotaciones de estos datos, pero además de eso, pudieron buscar información adicional, con el objetivo de fundamentar lo mejor posible sus argumentos. De esta manera, previamente al debate, leyeron artículos científicos (Vilches y Gil Pérez, 2008), buscaron en Internet y consultaron el libro de texto.

Tras llevar a cabo la búsqueda de información, cada grupo hizo una puesta en común. Tras ello, se realizó el debate en una sesión de 45 minutos. En esta sesión se estableció como única norma escuchar cuando otro compañero habla y no interrumpir, siendo el papel del profesor ejercer como moderador del debate.

\section{Método de investigación}

Se realizaron grabaciones en vídeo y audio del debate y se transcribió su contenido. Su análisis se llevó a cabo tomando como referencia el método 2 propuesto por Erduran, Simon y Osborne (2004) y basado en el modelo de argumentación de Toulmin (Toulmin, 1958). Dichos autores utilizan su método 2 para analizar la calidad de la argumentación solo en cuanto a las refutaciones, en este caso, hemos adaptado y aplicado su marco analítico de manera que contemplamos todos los tipos de argumentos distinguidos por Solbes, Ruiz y Furió (2010): datos, justificación, razones, fundamentación, refutaciones u objeciones y conclusiones. A partir de las relaciones que los alumnos establecen entre ellos (ver Figura 1), definimos un marco analítico que nos ha permitido evaluar la calidad de la argumentación.

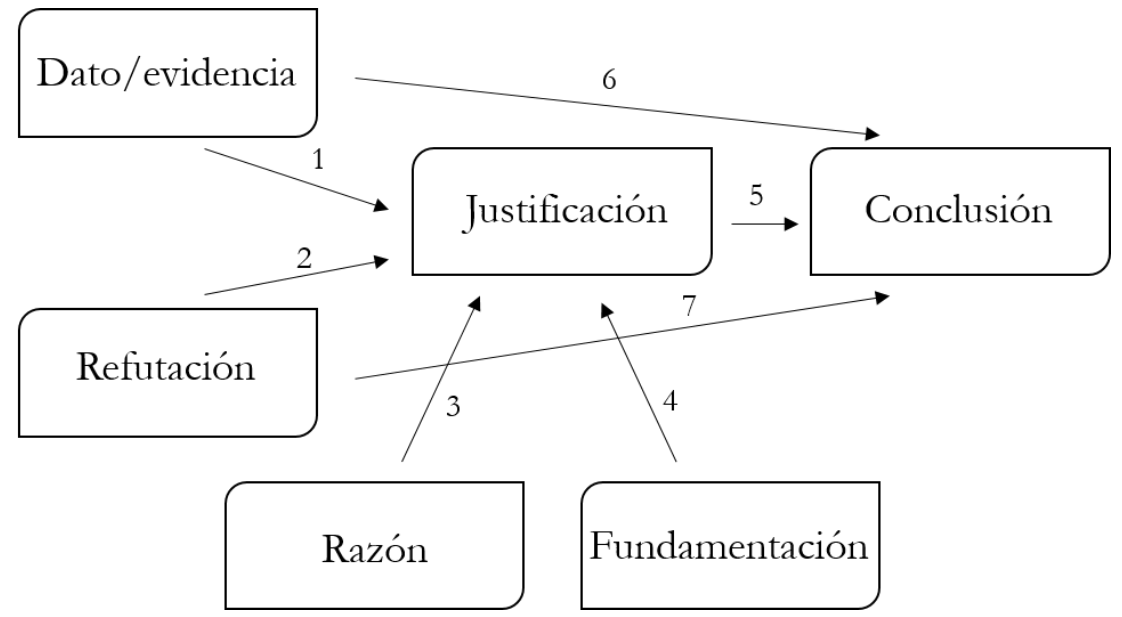

Figura 1. Posibles itinerarios a seguidos en la argumentación.

Por lo tanto, se establecen los niveles que aparecen en la Tabla 1. El nivel 1 correspondería a argumentos de calidad inferior y los de nivel 6 serían los de máxima calidad en este estudio. Se evalúa el nivel conseguido en cada una de las intervenciones de cada equipo y teniendo esto en cuenta, se establece el vencedor del debate. 


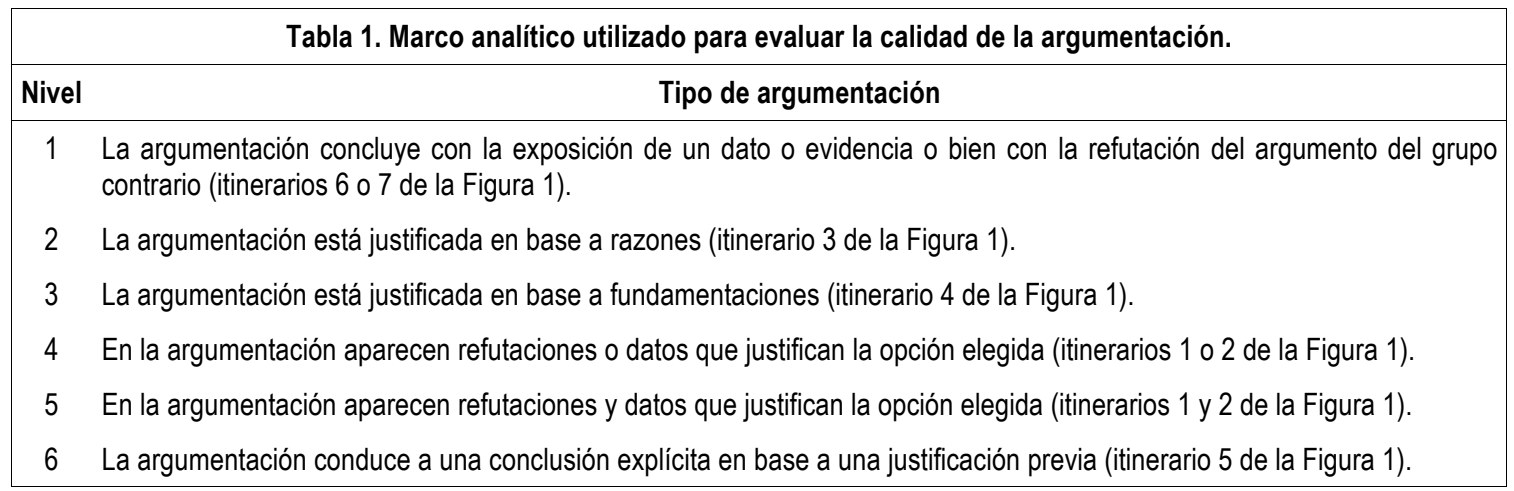

\section{Resultados}

A continuación, se muestran los resultados del análisis de las transcripciones de los vídeos y audios del debate, objeto de esta investigación.

Se ha analizado cada una de las intervenciones en el debate, identificando el itinerario argumentativo seguido. Se han contado 52 intervenciones en total, 32 intervenciones a favor y 20 en contra de la energía nuclear. De todas ellas, se ha estudiado el tipo de argumentos. En algunas intervenciones se seguían varios itinerarios para justificar la elección, y otras intervenciones se apoyaron en un solo tipo de argumentos. Por ejemplo: "si quitan la energía nuclear, muchos países como Francia se quedan sin energía ya que es el $23 \%$ de la energía consumida en la Tierra".

En esta argumentación, se utiliza un dato y una fundamentación. En la Tabla siguiente (Tabla 2) se muestran el número neto de veces que aparecen cada tipo de argumentos relativos a los fijados en la Figura 1.

\begin{tabular}{|lccccccc|}
\hline Tabla 2. Número de itinerarios seguidos en cada intervención \\
\hline & \multicolumn{7}{c|}{ Itinerario relativo a la Figura $\mathbf{1}$} \\
\cline { 2 - 8 } & $\mathbf{1}$ & $\mathbf{2}$ & $\mathbf{3}$ & $\mathbf{4}$ & $\mathbf{5}$ & $\mathbf{6}$ & $\mathbf{7}$ \\
A Favor & 14 & 2 & 14 & 17 & 0 & 0 & 0 \\
En Contra & 7 & 3 & 10 & 4 & 0 & 0 & 0 \\
\hline
\end{tabular}

Se observa que, en primer lugar, el equipo que expone sus motivos a favor de la energía nuclear, utiliza en cada una de sus intervenciones un mayor número de argumentos, ya que en un total de 32 intervenciones expresan 47 argumentos, mientras que el equipo que está en contra de la energía nuclear expone un total de 24 argumentos a lo largo de sus 20 intervenciones. Por otro lado, es llamativo que en ninguna de las intervenciones concluyeran, ni justificando su argumento, ni utilizando los datos o refutaciones, es decir, los alumnos no concluyen en ninguno de los casos. Ambos equipos utilizaron en mayor medida datos, evidencias y razones para tratar de justificar sus posturas y además el equipo que estaba a favor de la energía nuclear utilizó fundamentaciones para ello.

Tras analizar los niveles de calidad de estas argumentaciones, los resultados se muestran en la Tabla siguiente (Tabla 3). Se ha calculado el porcentaje en relación al número de itinerarios seguidos. 
Tabla 3. Porcentaje de argumentos que alcanzan cada uno de los niveles de calidad de la argumentación definido en la Tabla 1

\begin{tabular}{lcccccc|}
\hline & 1 & $\mathbf{2}$ & $\mathbf{3}$ & $\mathbf{4}$ & $\mathbf{5}$ & $\mathbf{6}$ \\
\cline { 2 - 7 } A Favor & 0 & 30 & 36 & 34 & 0 & 0 \\
En Contra & 0 & 38 & 16 & 38 & 8 \\
\hline
\end{tabular}

Ninguno de los dos grupos emite argumentos de calidad 6. El grupo defensor posicionado en contra de la energía nuclear es el único que expresa argumentos de nivel 5, es decir en una misma intervención aporta datos y refuta los argumentos contrarios. Y esto lo realiza en dos ocasiones (un $8 \%$ de los argumentos expresados). Por ejemplo, en la frase: "lo que dices no es cierto porque los residuos nucleares son peligrosos para el medio ambiente".

Por el contrario, el grupo que está a favor, no realiza ninguno. En cuanto a argumentos de calidad 4, ambos grupos están bastante igualados, y algo similar ocurre con los argumentos de calidad 2. Sin embargo, hay una diferencia llamativa en los argumentos de calidad 3. Un $36 \%$ de los argumentos que emite el grupo que está a favor del uso de la energía nuclear son de nivel 3 de calidad, mientras que solo un $16 \%$ de los argumentos que emite el grupo que está en contra son de este nivel. De manera comparativa, este último grupo recurre más a menudo a razones que a fundamentaciones para justificar su postura.

\section{Discusión}

A través de esta actividad se ha potenciado la competencia argumentativa de los alumnos. Además de ello, se ha establecido una categorización de los argumentos recogidos para intentar evaluar dicha competencia. Evaluar las competencias científicas no es fácil. Tal y como proponen Crujeiras y Jiménez Aleixandre (2015) lo más apropiado es evaluarlas mediante la puesta en práctica de las mismas. Por esa razón, en este trabajo se ha evaluado la competencia argumentativa oral a través de la exposición de argumentos en un debate en clase, para justificar la postura a favor o en contra del uso de la energía nuclear.

Para establecer un vencedor del debate se han analizado los argumentos expresados verbalmente por cada equipo, en base a los niveles de calidad previamente establecidos. Una de las conclusiones principales es que ninguno de los dos equipos está acostumbrado a argumentar de manera oral, por lo que no han sabido concluir en ninguna de sus intervenciones. Pese a eso, el grupo defensor de la energía nuclear ha verbalizado muchos más argumentos en el mismo periodo de tiempo que el grupo en contra. Además de ello, en varias intervenciones son capaces de aportar varios tipos de argumentos, llegando a dar 14 datos concretos y 17 fundamentaciones. Frente a esto, el grupo en contra de la energía nuclear aporta 7 datos y solo 4 fundamentaciones. Basándonos solo en esto, cabría dar la victoria al grupo a favor del uso de energía nuclear, sin embargo, en el presente estudio se ha llevado el análisis de los datos un paso más allá. Se han establecido niveles (6 niveles) para evaluar la calidad de las argumentaciones. Y estos niveles se han establecido en función de los itinerarios que siguen las argumentaciones de los participantes en el debate. En base a ello, se han calculado los porcentajes de cada nivel de calidad. Teniendo en cuenta esto, cabe concluir que el equipo en contra del uso de la energía nuclear es el único que manifiesta argumentos de calidad 5, por lo tanto, argumenta con un nivel de calidad superior al grupo a favor. De igual modo, se puede decir, que de manera neta sus argumentaciones se basan más en fundamentaciones que en razones lo cual aumenta el nivel de calidad de sus argumentos, existiendo además en sus intervenciones, un número mayor de refutaciones ante los argumentos del otro grupo. Por lo tanto, tomando como base el criterio establecido, el equipo en contra del uso de la energía nuclear debería ser el vencedor del debate. 


\section{Referencias}

Crujeiras, B. y Jiménez Aleixandre, MP. (2015). Análisis de la competencia científica de alumnado de secundaria: respuestas y justificaciones a ítem de PISA. Revista Eureka sobre Enseñanza y Divulgación de las Ciencias, 12(3), 385401.

Ferrés, C., Marbá, A. y Sanmartí, N. (2014). Trabajos de indagación de los alumnos: instrumentos de evaluación e identificación de dificultades. Revista Eureka sobre Enseñanza y Divulgación de las Ciencias, 12(1), 22-37.

Barmby, P., Kind, PM., \& Jones, K. (2008). Examining changing attitudes in secondary school science. International Journal of Science Education, 30(8), 1075-1093.

Campanario, JM. y Moya, A. (1999). ¿Cómo enseñar ciencias? Principales tendencias y propuestas. Debates y argumentación en las clases de física y química. Enseñanza de las Ciencias, 17(2), 179-192.

Driver, R. Newton, P \& Osborne, J. (2000). Establishing the norms of scientific argumentation in classroom. Science Education, 84, 287-312.

Erduran, S., \& Jiménez Aleixandre, M.P. (2007). Argumentation in science education: perspectives from classroom-based research. Dordrecht. Springer.

Erduran, S., Simon, S., \& Osborne, J. (2004). TAPing into argumentation: developments in the application of Toulmin's argument pattern for studying science discourse. Science Education, 88, 915-933.

George, R. (2006). A cross-domain analysis of change in students' attitudes toward science and attitudes about the utility of science. International Journal of Science Education, 28(6), 571-589.

Gil-Flores, J. (2012). Actitudes del alumnado español hacia las ciencias en la evaluación PISA 2006. Enseñanza de las Ciencias, 30(2), 131-152.

Jiménez Aleixandre, MP. (1998). Diseño curricular: indagación y razonamiento con el lenguaje de las ciencias. Enseñanza de las Ciencias, 16(2), 203-216.

Jiménez Aleixandre, MP. y Díaz de Bustamante, J. (2003). Discurso de aula y argumentación en la clase de ciencias: cuestiones teóricas y metodológicas. Enseñanza de las Ciencias, 21(3), 359-370.

Ruiz, JJ., Solbes, J. y Furió, C. (2013). Debates sobre cuestiones sociocientíficas. Una herramienta para aprender física y química. Alambique Didáctica de las Ciencias Experimentales, 64, 32-39.

Sardá Jorge, A. y Sanmartí Puig, N. (2000). Enseñar a argumentar científicamente: un reto de las clases de ciencias. Enseñanza de las Ciencias, 18(3), 405-422.

Solbes, J., Montserrat, R. y Furió, C. (2007). El desinterés del alumnado hacia el aprendizaje de la ciencia: implicaciones en su enseñanza. Didáctica de las Ciencias Experimentales y Sociales, 21, 91-117.
Solbes, J., Ruíz, JJ. y Furió, C. (2010). Debates y argumentación en las clases de física y química. Alambique Didáctica de las Ciencias Experimentales, 63, 65-75.

Toulmin, S. (1958). The uses of argument. Nueva York. Cambridge University Press.

Vilches, A. y Gil Pérez, D. (2008). La sostenibilidad y el debate nuclear. Revista Eureka sobre Enseñanza y Divulgación de las Ciencias, 5(1), 94-99. 